\title{
Ein analytisches Modell für die Blutdruckübertragungsstrecke der invasiven Blutdruckmessung mit externer Flüssigkeitskopplung
}

\author{
Wendt, Z.; Kraft, M.; Boenick, U. \\ Institut für Mikrotechnik und Medizintechnik, Technische Universität Berlin
}

\section{EINLEITUNG}

Die invasive Blutdruckmessung spielt in der Anästhesie und in der Intensivmedizin eine wichtige Rolle. Im klinischen Alltag wird vorwiegend das preisgünstige extrakorporale Blutdruckmeßverfahren mit einem externen Einwegtransducer eingesetzt. Die Qualität dieses Verfahrens hängt im wesentlichen von dem Druckübertragungsverhalten der Meßstrecke ab, welche hauptsächlich aus dem Katheter, den Verlängerungsschläuchen, der Flüssigkeitssäule und dem Transducer besteht. Zusätzlich beeinflussen in der Flüssigkeitssäule vorhandene Luftblasen die Druckübertragungsqualität. In diesem Beitrag wird eine analytische Methode zur Bestimmung der Übertragungseigenschaften dieses Blutdruckmeßsystems anhand eines erweiterten neuen Modell vorgestellt. In ihm sind alle wichtigen Bestandteile der Druckübertragungsstrecke hinsichtlich ihrer Geometrie und Materialeigenschaften berücksichtigt.

\section{METHODE}

Die Blutdruckübertragungsstrecke läßt sich als ein kontinuierliches mechanisches Schwingungssystem betrachten, wobei der pulsierende Blutdruck den Schwingungserreger darstellt. Wie jedes schwingfähiges System kann auch diese Übertragungsstrecke durch die zwei Kenngrößen, Eigenfrequenz und Dämpfung, beschrieben werden. Aus ihnen lassen sich wichtige Folgerungen auf die Blutdruckübertragungseigenschaft ableiten. Für die analytische Behandlung dieses Problems wurde das im $A b b . I(b)$ gezeigte Modell zugrunde gelegt. Das reele System $(A b b . I(a))$ ist in der Modelldarstellung vereinfacht in n-Teilsysteme unterteilt. Somit kann das kontinuierliche Schwingungssystem durch ein in $A b b . I$ (c) dargestelltes diskretes $\mathrm{n}$-faches Schwingungssystem angenähert werden.
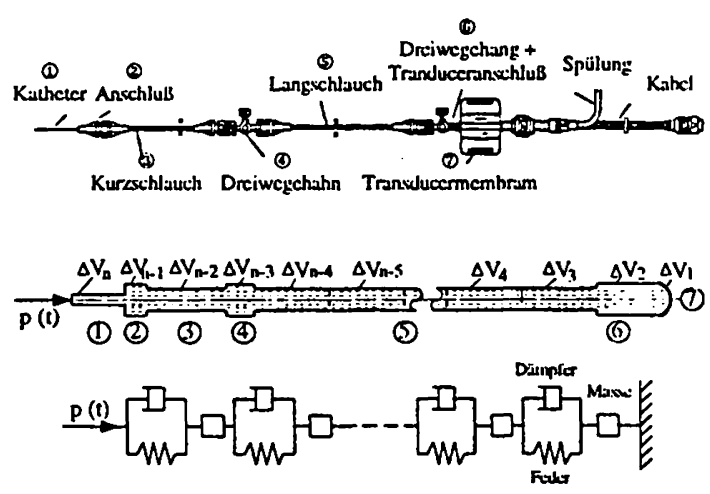

Abb. 1:(a) ein reeles blutdruckmeßset, (b) das Ersatzmodell, (c) das dem Modell entsprechende nfache Schwingungssystem.
Die Ermittlung des Druckübertragungsverhaltens über die Kenntnis der Eigenfrequenz und Dämpfung geht von der Aufstellung systemspezifischer Bewegungsgleichungen des Modells aus. Dabei wurde die analytische Methode nach Lagrange vorgezogen, da diese auf einer Berechnung der Energieausdrucke des Gesamtsystems basiert, wodurch die komplizierten Reaktionskräfte zwischen den Teilsystemen übergangen werden.

Die Lagrangesche Bewegungsgleichung läßt sich allgemein wie folgt ausdrücken:

$$
\frac{d}{d t} \partial T_{\text {ges. }} \partial \frac{\partial T_{\text {ges }}}{\partial x_{i}}+\frac{\partial U_{\text {ges }}}{\partial x_{i}}=K_{i} \quad(i=1,2, \ldots f)
$$

( $x_{i}$ : die verallgemeinerten Lagekoordinaten,

$f:$ Anzahl der Freiheitsgrade,

$T_{\text {ges: }}$ die gesante kinetische Energie des Schwingungssystems,

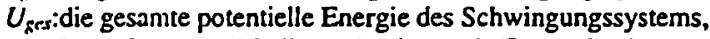

$K_{i}$ : Dämpfungs- und Reibungskräfte sowie Erregerkräfte.

Als verallgemeinerte Lagekoordinaten des Schwingungsmodells wurde die durch den Erregerdruck verursachte Innenvolumendehnung $\Delta V_{i} \quad(i=1,2, \ldots n)$ jedes Teilsystems definiert. Die kinetische Energie, die potentielle Energie und die virtuellen Arbeiten der Reibungsund Druckkräfte durch die virtuelle Volumendehnung ließen sich als Funktionen von $\Delta V_{i}$ herleiten. Dabei wurde die Theorie eines kreiszylinderischen Rohres [2] für eine Schlauchstrecke, die Kirchhoffsche Plattentheorie [2] für die Transducermembran verwendet. Die Bewegung des Füllfluides wurde als laminar angesehen und für die Berechnung der Flüssigkeitsreibung wurde näherungsweise das Hagen-Poiseuillesche Gesetz eingesetzt. Die Herleitung der virtuellen Arbeit der inneren Reibung einer Kunststoffschlauchstrecke aufgrund einer virtuellen Schlauchdehnung basierte auf einer Berechnung der Kunststoffverlustarbeit [3].

\section{ERGEBNIS}

Die hergeleiteten Lagrangeschen Bewegungsgleichungen des Blutdruckübertragungsmodells lassen sich in einer Vektordifferentialgleichung zweiter Ordnung zusammenfassen, die ein mehrfaches gewöhnliches Schwingungssystem kennzeichnet:

$$
M \ddot{x}+P \dot{x}+Q x=p(t)
$$

es gilt:

- M, P, Q: nxn- symınetrische Massenmatrix, Dämpfungsmatrix und Federnnatrix: $x, p(t)$ : Vektordarstellung der verallgemeinerten Lagekordinaten und des Erregerdruckes.

- Die Elemente der Matrizen M, P, Q sind Funktionen der Geometrie- und Materialkenngröße der Teilsysteme. Aufgrund des Umfangs dicses Beitrages werden sie hierbei nicht explizit dargestelle. 


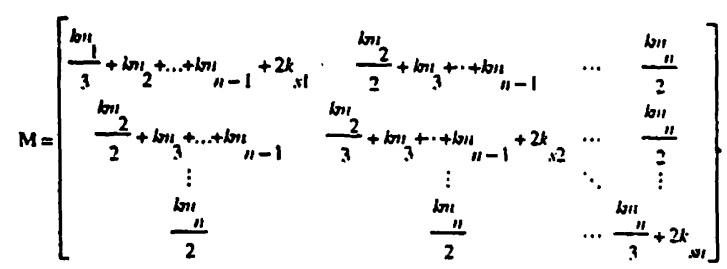

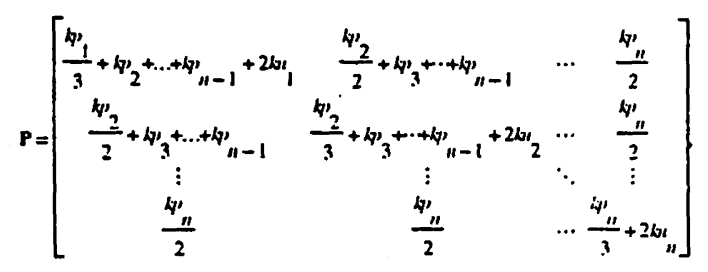

$Q=\left[\begin{array}{cccc}{ }_{M 1} & 1 & \cdots & 0 \\ 0 & c_{S 1} & \cdots & 0 \\ \vdots & \vdots & \ddots & \vdots \\ 0 & 0 & \cdots & c_{S 1}\end{array}\right] \quad x=\left[\begin{array}{c}\Delta V_{1} \\ \Delta V_{2} \\ \vdots \\ \Delta V_{11}\end{array}\right] \quad P(0)=\left[\begin{array}{c}\mid x(t) \\ 1(x) \\ \vdots \\ \mid(t)\end{array}\right]$

Nach der Überführung der Gl.(2) in die Zustandsgleichung nach [1] wurde die Systemmatrix A des Ersatzmodells ermittelt:

$$
A=\left[\begin{array}{cc}
0 & E \\
-M^{-1} Q & -M^{-1} P
\end{array}\right]
$$

(0: $n \times n$ - Nullmatrix, E: $n \times n$ - Einheitsmatrix)

Aus den Eigenwerten der Systemmatrix A

$\lambda_{i}=\delta_{j} \pm i \omega_{j}, \delta_{j}<0 \omega_{j} \neq 0, i=1,2 \cdots 2 n, j=1.2, \cdots n$

können dann die Eigenfrequenzen $\omega_{j}$ des Modells direkt abgelesen werden. Die Dämpfungen des Ersatzmodells lassen sich wie folgt berechnen:

$$
v_{j}=\frac{-\delta_{j}}{\sqrt{\delta_{j}^{2}+\omega_{j}^{2}}}
$$

Wobei in erster Linie die erste Eigenfrequenz und die dazugehörige Dämpfung von Interesse sind.

Die Berechnung erfolgte mit Hilfe einer Software zur Lösung mathematischer Problemstellungen.

Ein Vergleich der Berechnungsergebnisse mit den MeBergebnissen des Sinustests, ein Meßverfahren zur empirischen Ermittlung der Eigenfrequenz und Dämpfung (beschrieben in [4]), mit einer Reihe von Testsystemen ergab eine gute Übereinstimmung der berechneten ersten Eigenfrequenzen mit den Meßwerten und eine tendenzielle Aussagefähigkeit der berechneten Systemdämpfung beim Variieren eines Parameters, wie z. B. Kathetertyp oder Schlauchlänge. $A b b .2$ enthält die graphische Darstellung der $\mathrm{Me} B$ - und Berechnungswerte einer Beispielreihe von 12 Testsystemen mit einem Transducer und einem PE-Schlauchset mit den folgenden Abmessungen:

Außen-/Innendurchmesser der Schläuche: $2 / 1 \mathrm{~mm}$,

Kurzschlauchlänge: $15 \mathrm{~cm}$ für alle Testsystemc,

Langschlauchlänge: $100,150,200 \mathrm{~cm}$,

und jeweils vier Teflon-Kather: 22G/80, 18G/160, 20G/80, 20G/160.
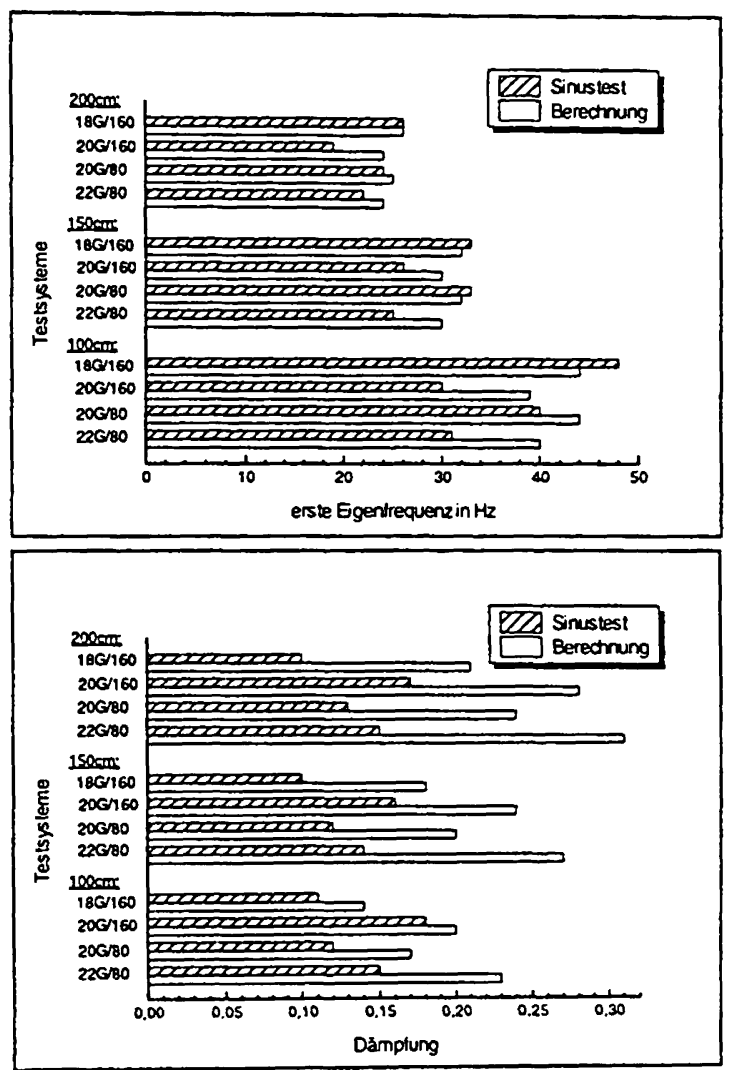

Abb. 2: Berechnungs- und Meßergebnisse (Sinustest) einer PE-Testreihe mit 12 Testsystemen.

\section{DISKUSSION}

Anhand des vorgestellten Berechnungsverfahrens läßt sich die Frage klären, in wie weit sich die erste Eigenfrequenz und die Dämpfung ändern, wenn Struktur oder Parameter der Meßstrecke varriert werden.

Nachfolgend aufgelistete Problemstellungen sind weitere Beispiele, die mit Hilfe des beschriebenen Modells analysiert werden könnten:

- Einfluß der Lage und Größe einer Luftblase in der Flüssigkeitssäule,

- Variation bestimmter Parameter der Meßstrecke,

- Zwischenschalten von Manipulationseinheiten serieller, paralleler oder verzweigter Anordnung.

Da diese analytische Methode auf die Einzelheiten der Meßstrecke eingeht, paßt sich die Berechnung beliebig den Randbedingungen der Meßstrecke an. Mit Hilfe des vorgestellten Verfahrens lassen sich künftige Entwicklungen bzw. Optimierungen hinsichtlich ihrer Beeinflussung des Ühertragungsverhaltens bereits im Vorfeld rationell prüfen.

\section{LITERATURVERZEICHNIS}

[1] P.C. Müller: „Lineare Schwingungen“, Akademische Verlagsgesellschaft, Wiesbaden 1976

[2] I. Szabò: „Höhere Technische Mechanik”, 5. Auflagc, Springer Verlag 1985

[3] H. Sclimiedel: „Kunststoffprüfung“, Hanser Verlag 1992

[4] N. Mendler. „Blutdruckmessung mit Einweg. Sensoren", "Kardiotechnik" 11. Jahrgang/Hef $3 / 1988$ 